\title{
SATSEARCHTORY: Searching Online Platform for Niche Product in a Metropolitan Area
}

\author{
Sutipong Sutipitakwong and Pornsuree Jamsri* \\ Faculty of Information Technology, King Mongkut's Institute of Technology \\ Ladkrabang, Bangkok, Thailand \\ E-mail: 61070369@it.kmitl.ac.th; pornsuree@it.kmitl.ac.th \\ ${ }^{*}$ Corresponding Author
}

Received 25 April 2020; Accepted 05 June 2020;

Publication 17 August 2020

\begin{abstract}
Satsearchtory helps users perform an uncomplicated search for products that are rare items. It also can be used for nonrare or regular items by showing locations for purchasing the item and comparisons of prices and promotions that are available for ensuring the best deal. The tool, thereby, can guarantee users' satisfaction through online service using search engines for accurate results. Because search engines using keywords and phrases have not been able previously to achieve a $100 \%$ result for users, Satsearchtory was designed to increase the percentage of users' satisfaction.
\end{abstract}

Keywords: Online community, commercial platform, search engine, niche product, metropolitan area.

\section{Introduction}

Individuals with a shared interest frequently look for various groups and connections with similar interests to form a community. Often this is the case so that they can exchange, buy, or sell products and services that fit their similar needs or requirements. Specifically, communities may share an

Journal of Mobile Multimedia, Vol. 16_1-2, 85-102.

doi: 10.13052/jmm1550-4646.16125

(C) 2020 River Publishers 
interest out of the ordinary. Thus, when searching for an extraordinary item, such as obsolete technology parts and out-of-date automobile spare parts their search may prove futile by returning no results. Even if on the rare occasion persons may sometimes find the items they need locally, the cost often includes a high ceiling price extra fees as valued-added to rare items.

This is typically encountered in densely populated metropolitan locations. Bangkok, thus, was selected as the example for this pilot research for two primary reasons. First, Bangkok has a high density of approximately 10 million people living in 605.7 square miles-critically important to reflect the scope of a large community with extraordinary needs and interests. Second, Bangkok's environment is intense with traffic congestion during rush hours. It requires a great deal of travel time to drive from one place to another-home to work and back-for the lunch hour and retail shopping. [6, 8, 14, 15]

This research focuses on the factors that impact searching for rare items. Satsearchtory is a cutting-edge technology to gather and filter accurate search results to guarantee users' satisfaction.

\subsection{Objectives}

In this research, our primary goal is to identify key factors and keywords used on any web-based search engine that offers effective search results. The goal is to collect and understand users' actions in searching for specific items. Data collected will assist the next step of developing a new tool that would work effectively for people in the metropolitan Bangkok area to find desired products and services with greater ease.

\subsection{Target Group in Metropolitan Area (Bangkok)}

The target group for this website is users who are interested in the niche market and are in the metropolitan Bangkok area. A niche market focuses on specific items of each category that interests a small number of people who are looking for a certain item in their field of interest such as limited

Table 1 Example parameters of experiments

\begin{tabular}{ccccc}
\hline & & \multicolumn{3}{c}{ Thailand Platform } \\
Feature & CraigList & Lazada & Shopee & Tarad.com \\
\hline Basic Info & $\checkmark$ & $\checkmark$ & $\checkmark$ & $\checkmark$ \\
Community Market & $\checkmark$ & $\checkmark$ & $\checkmark$ & $\checkmark$ \\
Buy Now or Later & - & - & - & - \\
User Review Rating & $\checkmark$ & $\checkmark$ & $\checkmark$ & $\checkmark$ \\
\hline
\end{tabular}


technology items and discontinued or rare items by narrowing down the market to specific individuals who are committed to finding or listing these items $[1,4]$.

\section{Literature Review}

\subsection{Features}

Current online stores and services utilize various categories of listings to search, find, buy, and sell products within the same group or range. The interface contains the following features to aid users:

- Basic information: each item will have its name and its description, what it does, and/or its use. This is generally a standard requirement for all items on commercial websites. This feature is critical for users in deciding whether the item meets their required needs [4].

- Community market: a feature enabling users to list their items and products for sale. This lets users sell their items through the website and for users as buyers to compare similar items in order to find the best item(s) for their needs [2].

- Users' reviews: a feature that allows previous owners of item(s) to provide feedback and comments on their purchased item as to whether it worked well or not. This feature assists buyer/users to better decide whether a product is worth purchasing or not $[5,7]$.

- Price comparison and promotions between stores with the same item: this feature lets users focus on sellers with the best deal when the desired item is available in different stores. The application will select and display the best deals in the specified area for users to make a selection [18].

\subsection{Existing Platforms}

The website utilizes three platforms, namely the Steam Community Market, Craigslist, and PricePanda. Features such as the market area and item information are on Craigslist with a graph illustrating price and rate fluctuations from the Steam Community Market [2, 3].

- Steam Community Market: offers an online marketplace for users to check prices for selling or buying. Each item has a graph displaying its prices and fluctuations [2].

- Craigslist: offers a wide variety of items posted online with each user of the website able to contact the seller and buy an item individually. 
Craigslist has item descriptions as categories of each item that falls within [3].

- PricePanda: an online platform for online shopping that enables users to compare items in the same category mainly by showing the best price, average price, followed by all the prices of an item [18]. Likewise, some product owners provide the function "compare price" but this happens within their products model in the same company such as Apple provides "compare price" within the same product line iPhone or MacBook among various models.

\subsection{Related Technology}

Most users tend to search for products through web-based platforms or apps on a mobile device. Because these channels are easily accessible, users usually choose a most convenient platform. Use of web-based platforms and apps is the most popular choice for searching.

\section{Web-based platform}

- The advantages of this platform allow users to get a higher rate of satisfaction depending on their keyword searches.

- Some results may only support a web platform for display.

- Results are not limited to items made available only by the apps.

- Given that there are over 8 million people in Bangkok it is estimated that at least 1/4 (2 million) would be using this tool [14].

- Users are able to search for items freely and more accurately.

- Users are able to search for regular items or niche market items according to their preference, for example, car parts and technology gadgets.

\section{Apps on a mobile device}

- Online stores might not offer a 100\% satisfactory search with an add-on to the store that helps filter out searches [9].

- Add-ons could be provided to apps such as Lazada, Ali Express, Amazon, and Shopee.

- Apps provide categories and subcategories of various products.

- People all over Bangkok use apps such as Lazada every day; so, this makes sense that this tool would be helpful in making searches $[12,14,15]$. 
- Incorporates techniques used in Pricepanda and adapts it for a wider range of usage opportunities [18].

\subsection{Communication Channels}

- Email: most web services utilize email for contacts between the customer and the seller through the service platform of providers. The service platform providers are intermediaries between buyer and seller.

- Social media (SM): another popular method used for selling and buying. In Thailand, the number of SM users, such as Facebook, numbered approximately 26.3 million in 2018 and is expected to reach 26.9 million in 2019. From a wide range of individuals to business sellers, people in Thailand use SM a great deal to access online stores and gain information on sale items from vendors [17].

- Instance messaging (IM): People are familiar with purchasing online through IM such as LINE chat. It provides prompt reply and sufficient features to verify the user and buyer, sending images after making payment as evidence of payment to the seller. It has become a popular new way of online shopping. Some buyers access through LINE Ads that link them to the store's item and customer easily as though visiting the store's website with a list of items, location, and contact info in one place.

\subsection{The Gap of the Current Situation}

The feature "Buy Now or Buy Later" is not available on any online platform. This new feature in Satsearchtory utilizes a database comparing previous prices and trends to current prices by graphs depicting the changing rate of the items that can be used to make predictions about future prices. It is an essential feature helping users decide whether to buy a product immediately or at a later time.

In existing online market platforms basic information of the product, community market for listings, and user review ratings are available. The main missing feature, however, from these platforms that provide price comparisons and promotions for different stores, is that the user still needs the "Buy Now or Buy Later" feature after they select their best-suited item with the best price. "Buy now or Buy Later" further analyzes the trends of the promotion of the items in each store ensuring the best decision within the buyer's timeframe. 


\section{Design and Development}

The existing technology of many search engines and available platforms, such as Google search and online community markets, enables users to perform common steps or actions in searching for specific items. These actions are most often universal. For example, in Google search, when a person has a specific item in mind, he or she uses keywords to reach his or her goal. The number of searches each time and time spent on search are the vital variables that illustrate the satisfaction of each user. Thus, we can show the overview of the item search as Figure. 1.

\subsection{Key Findings}

- There is a specific market for niche goods that are very popular for searches in Thailand.

In understanding an item's popularity, Google trends in Figure 2(a) could be used to know the frequency of keywords used for searching location and generated topics related to a keyword. Furthermore, in Figure 2(b) there is a graph that shows the popularity of the searches over time. This information can be useful for the Satsearchtory tool to increase the possibility of a satisfactory search result, when it shows there is only a specific location having the item and a small number of related websites offering it. The value of an initial search is so much that the user searches more in specific places such as a niche market.

\subsection{Prototype}

The key findings lead to developing a prototype both with key features matching user needs and missing features to raise search satisfactory results

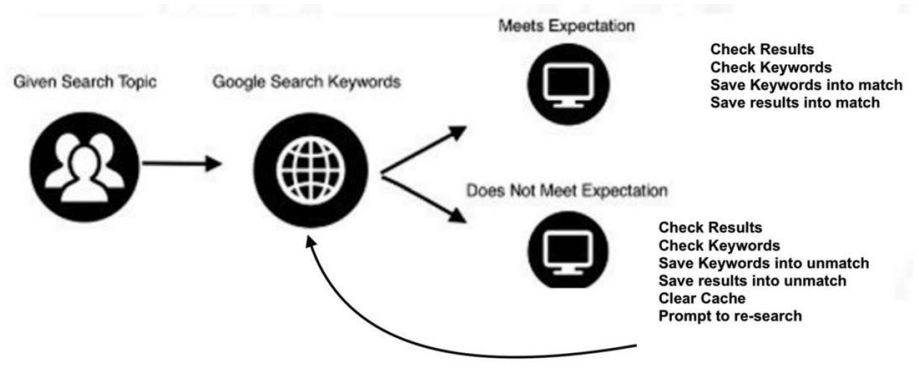

Figure 1 The overview of item search using results from Google trends. 


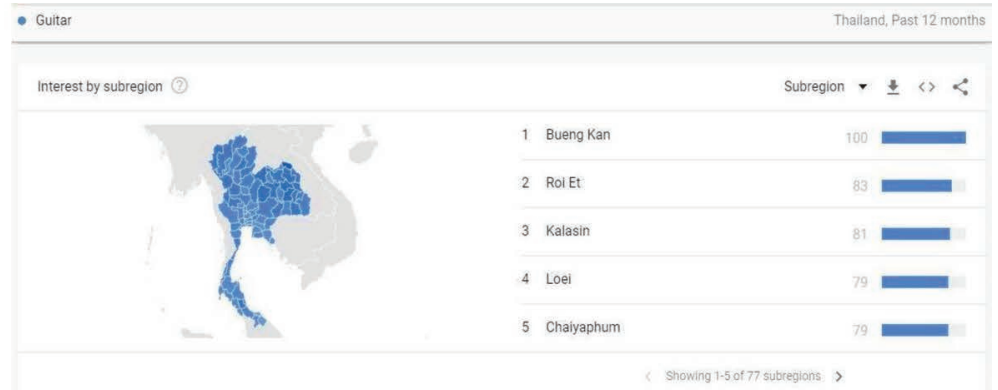

(a)

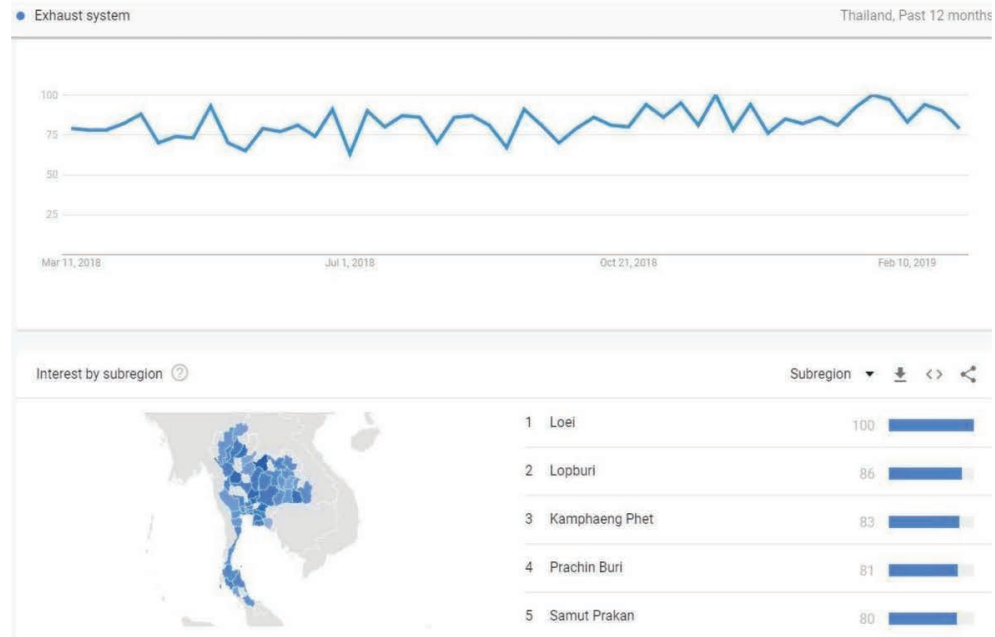

(b)

Figure 2 Google trends results on keywords.

of a low-demand item. To receive a response from the target group a prototype was created on a mobile platform. It was tested to see the effectiveness of this tool. Based on the research and findings, this prototype's goal is to attend to the gap between web-based platforms and mobile platforms. The intention is to focus on the strong points of the web-based platforms and turn those features into more compact, convenient, and easily accessible mobile features. Furthermore, from the prototype this research will dig down into the understanding of each feature of the tool in addition to the mechanics behind each feature. 


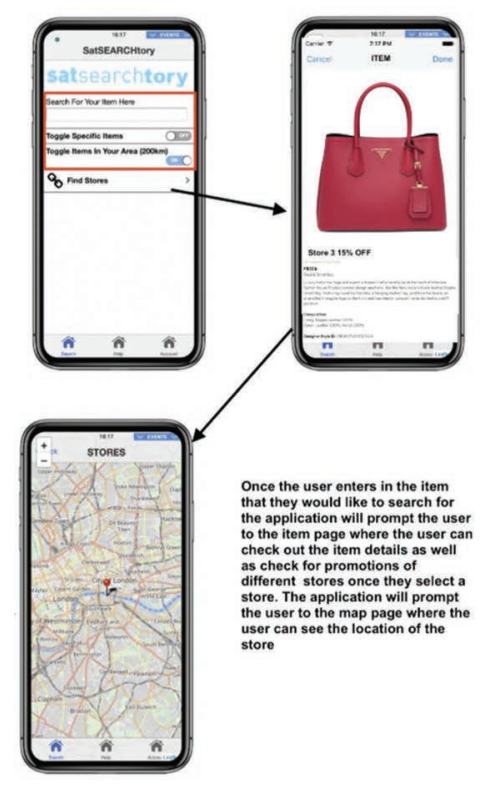

(a)

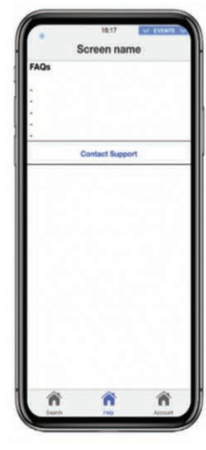

(b)

Figure 3 Initial prototype version.

Prototype in Figure 3 consists of features such as toggle search area, item's page, store searching on maps, support, and accounts pages. The navigation is facilitated easily through the app; it allows the users to maximize satisfaction degrees such as customizing the settings and changing some configuration regarding the requirements.

This prototype is an initial version based on the design and its simplicity with limited features and pages. The designed app only uses three main pages for operational usage. The search feature consists of a keyword input box, a wish list with a toggle section for specific items, and filtering out other items related to the searched keyword. As shown in Figure 3(a), the search feature takes the users to the item page where they can locate to a map page with marked store locations for the searched item. This allows users to select the nearest store with the item. Other pages referred to in Figure 3(b) are FAQ pages with an option to contact support and the user's account page. The FAQ page is self-explanatory and links with the user's account page for further contacting support staff. The user's account page aims to minimize the steps of registering and filling out any information as this design is geared toward 
simplicity. Reducing the steps of registration allows users an option of linking their Google account or their Facebook account. This will minimize the app's data and shorten steps to set up an account. These two options were selected because the two accounts were used by many students. Users generally have either one or both accounts.

Prototype in Figure 4(a) illustrates an iteration version, i.e., a newer version of the Satsearchtory mobile platform. While using most of the former styling components and main pages, version 2 removes unnecessary contents. It has more useful features than the previous prototype. This new version allows for the searching of items and stores to find the items that are best fit to their needs based on their cookies. The version 2 of the prototype shown in Figure 4(b) introduces the ratings of results for the satisfaction of users. It creates a better understanding of the algorithm showing cookies to ignore and cookies useful for best results. Besides finding stores with desired items, version 2 reviews promotions and discounts for the best deal among stores as

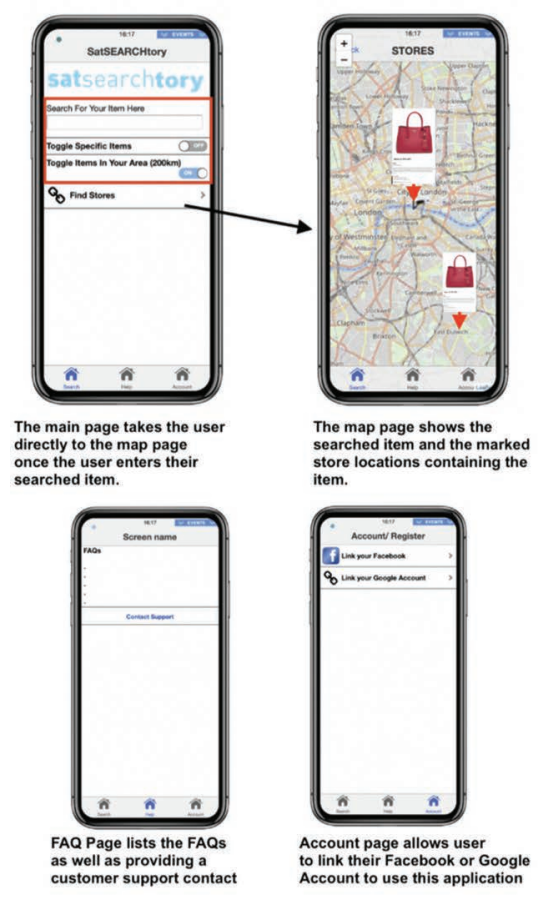

(a)

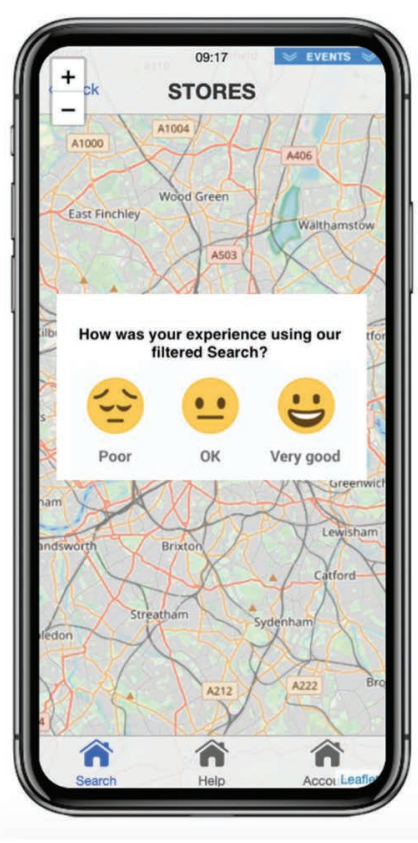

(b)

Figure 4 Prototype version 2. 
seen in price comparisons and locations. It allows the application to work in finding items in different stores and selecting the nearest desired store(s) with a promotion or discounted item.

Imperfections of the first prototype lead to development of version 2 . From Figure 4(a), it is shown that instead of the item page after the search it now directly shows the different stores with available items. Users can now click on the store for a popup dialogue to the reviews' ratings, product description, prices, and promotions offered rather than first the item page and then going to the store. This feature allows users to compare store locations, prices, and promotions of the store with more ease and accessibility. Maintaining the minimalistic design and concept help the account pages stay unchanged. In Figure 4(b), two additional features added to this version are rating of the desired results and promotional comparisons. The application manages to make adjustments to its database of the results and the entered keywords. The promotional comparison page allows users to select different stores for price comparisons and promotions that best meet their needs.

Figure 5 shows the page comparing available promotions of the desired item. It compares different stores with their prices and promotions and "Buy Now or Buy Later" features.

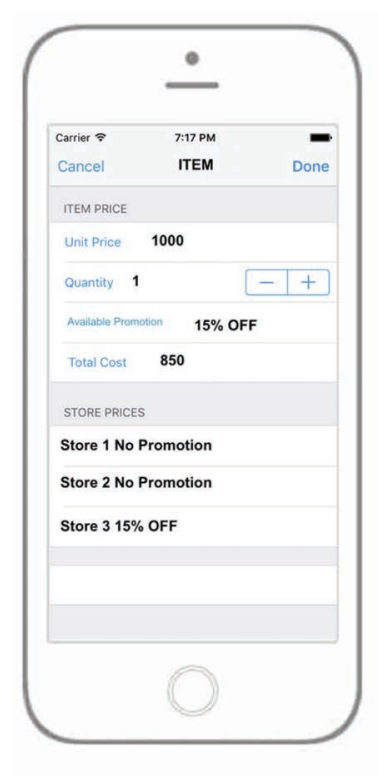

(a)

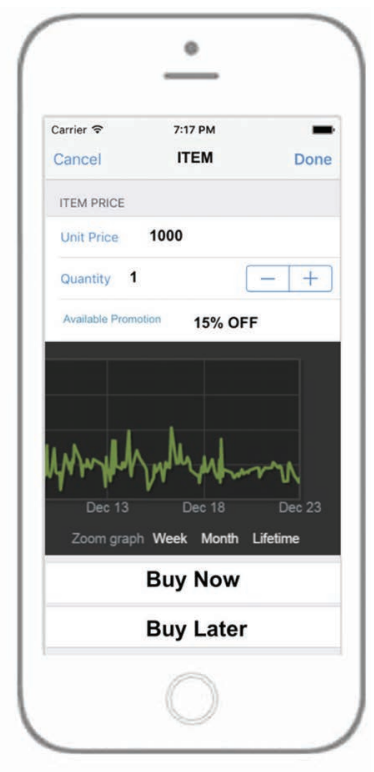

(b)

Figure 5 Understanding of promotions. 
Figure 5 is the comparison page example in a simple and minimalistic design to come to the point. The layout features the item's name, price, quantity, available promotions and "Buy Now or Buy Later," plus total cost and selected stores shown in the mobile phone's screen. The two main features of this page are the available promotions located in Figure 5(a) and "Buy Now or Buy Later" located in Figure 5(b). The available promotions alert the user regarding the best promotional deals during the current period. For example, store 1 might have a 5\% discount promotion and store 2 a $10 \%$ discount promotion. This feature alerts selection of store 2 as the best available promotion.

The "Buy Now or Buy Later" feature in Figure 4(b) is the main selling point of this application because it plugs in the main gap of the current situation. This section shows a graph of the item illustrating the prices in the past and predictions of the prices in the future. It allows users to review and decide which option is best for them. If the user selects the "Buy Now" feature, then the application automatically selects the best deal. If the user selects the "Buy Later" feature, then the item is added to the user's wish list and the user will then get a notification of a better promotion or price at a later time.

To further understand the satisfactory results according to the rating the user provides, the application will have to consider the following flow chart (Figure 6) that explains the process and algorithms the application manages and adjust to come up with satisfactory results.

This flowchart explains the basic algorithms the tool uses in its rating system to underscore how it shows the results. If the user is satisfied with the results, the tool will continue to show similar results. However, once the user rates a dissatisfied result the tool shows less results similar to what was shown previously and displays something new. This also helps with sorting out the algorithms of "Action item location" where the application can optimize to order these keywords and, thus, provide the most satisfactory results. Although some keywords inputted might not contain all provided categories, the application creates a notice of the rating and adapts it with the inputted item from the search. Finally, this application will use its concept of machine learning to fully understand which results to display and which results are incorrect.

\section{Results and Analysis}

After testing the results using tests on different browsers and clearing cookies the search methods' results are as follows: Selecting a topic and testing 


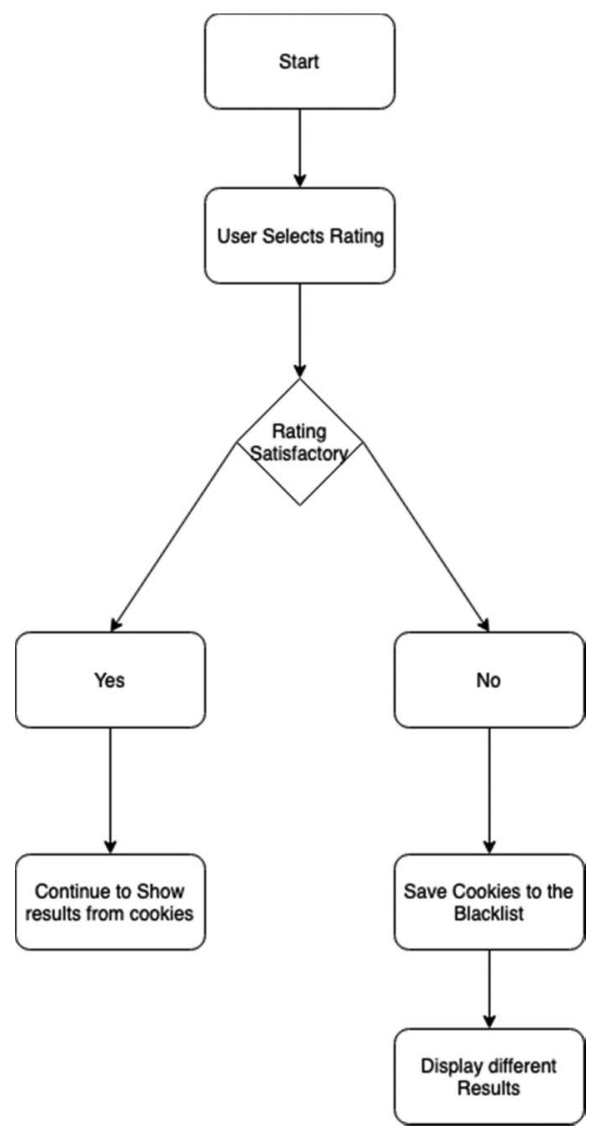

Figure 6 Comparison and understanding of cookies and ratings.

different keywords limited to three words to search for a specific item, results vary from $0 \%$ as in not matching the expected results at all to $100 \%$ in meeting expectations. However, these three words search enables more understanding of these items. Items deemed specific without many related searches will reach a higher range of percentage. Whereas the items with many related searches using the three words may not be enough to specify the item and the satisfactory result falls into the lower range.

Figure 7 demonstrates an example search of search results for a guitar showing the keywords used to search the results and the percentage of user satisfaction with the results. It is clearly shown that the keywords are very critical to the results of the search. In this example, the "Ibanez" brand has 


\begin{tabular}{|c|c|c|c|}
\hline Searches & Expectations & Results & Satisfaction \\
\hline $\begin{array}{l}\text { Buy Ibanez } \\
\text { Thailand }\end{array}$ & \multirow[t]{2}{*}{$\begin{array}{l}\text { Shows Ibanez } \\
\text { guitar models or } \\
\text { places to buy } \\
\text { "Ibanez" branded } \\
\text { guitars }\end{array}$} & 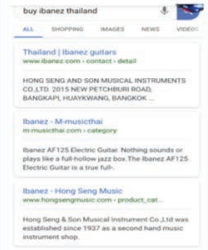 & $\begin{array}{l}100 \% \\
\text { Links show shops } \\
\text { to buy the guitars }\end{array}$ \\
\hline buy ibanez guitar & & 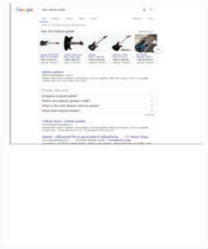 & $\begin{array}{l}80 \% \\
\text { Links show online } \\
\text { shops to the } \\
\text { guitars with an } \\
\text { addition of the } \\
\text { official ibanez } \\
\text { website which } \\
\text { does not sell the } \\
\text { guitars directly }\end{array}$ \\
\hline
\end{tabular}

Figure 7 Example search results from website.

\begin{tabular}{|c|c|c|}
\hline $\begin{array}{l}\text { Ibanez guitar } \\
\text { thailand }\end{array}$ & 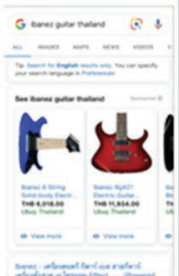 & $\begin{array}{l}70 \% \\
\text { Shows online } \\
\text { shops but does } \\
\text { not link to any } \\
\text { local dealer } \\
\text { music stores }\end{array}$ \\
\hline $\begin{array}{l}\text { Thailand } \\
\text { Ibanez }\end{array}$ & 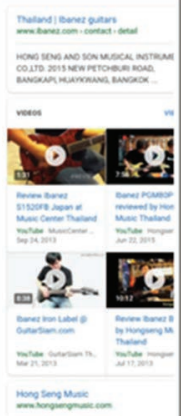 & $\begin{array}{l}20 \% \\
\text { Shows one } \\
\text { store to buy } \\
\text { the guitars the } \\
\text { rest are videos } \\
\text { and the } \\
\text { official ibanez } \\
\text { site which } \\
\text { does not } \\
\text { directly sell } \\
\text { guitars }\end{array}$ \\
\hline
\end{tabular}

Figure 8 Example search terms graph and results, percentage of satisfaction from keywords.

been used as a search term. Figure 8 clearly shows that if the words are specific enough by inputting the "action item location" then it can make for a satisfactory result. Whereas using the "action item" may result in a poorer result. Thus, setting up a standard for the application to change and fix the 
formatting of keywords is needed so that the user enters to fit "action item location."

The results illustrate that adding a key term could lower or increase the results that meets expectations and fulfills the level of satisfaction as in Figure 8. It could also mean that the wrong terms will lead to a nonsatisfactory result. The phrase "Wrong Terms" refers to terms that are too vague or have too many related results. The table above clearly shows that without the action in the keyword the satisfactory result can drop since the search engine cannot directly understand what the user wants to do with the item. On the other hand when the user inputs the location, then the item proves that the search engine is order sensitive and will output a different result by the order the words are inputted in. This can result in results that are completely off.

\section{Web-based platform}

- Wrong key terms could lead to irrelevant results.

- Specific key terms will show mostly relevant results.

- The key terms are still counted and it is usually unable to fulfill $100 \%$ of expectations by the customer.

\section{Conclusions and Future Research}

After reviewing the data samples, prototypes, and figures, consideration of the data collected from Google trends and tested search results clearly show that search engines will vary their results. This is dependent on the keywords and the location where users select to search for items. Furthermore, it is also evident that the keywords used are order sensitive with the best results obtained through the following layout "action item location." With this finding, the queries made may lead to the correct results or results that are completely off from the user's expectations dependent on the order the user inputs the keyword. With some items hardly appearing because of low demand leads to the conclusion that the search engines are still not capable of on point information that realizes the user's needs.

\subsection{Future Research}

Future research should focus on collection of more data by testing different keywords' inputs in different orders or to expand or lower the amount of words that can be used to further analyze it for creating an algorithm which 
is generated based on the actual data gathered from target groups. Cuttingedge technology will be used in the new platform that raises the satisfaction to meet expectations of users to a higher level and allows for a faster response time of gathering results and displaying them to the user. Furthermore, it is needed to understand the gathering of searches of Google's organic search algorithms to alter the ordered keywords to gain the most satisfactory result (Figure 9).

The flowchart in Figure 9 explains the basic algorithm of filtered Google searches for paid ads and organic searches. Organic searches are searches based on Google's algorithm for websites that do not pay for advertising based on the information validity of the website.

From the current research this flowchart has been created to provide a basic understanding of Google's organic search algorithms that can be used in further research. As this flowchart derives from the functionality of the application and how it gathers in results based on keywords, popularity, and compatibility, this flowchart can be used as a guideline for the basis of the understanding of Google's organic search algorithms. Since this flowchart

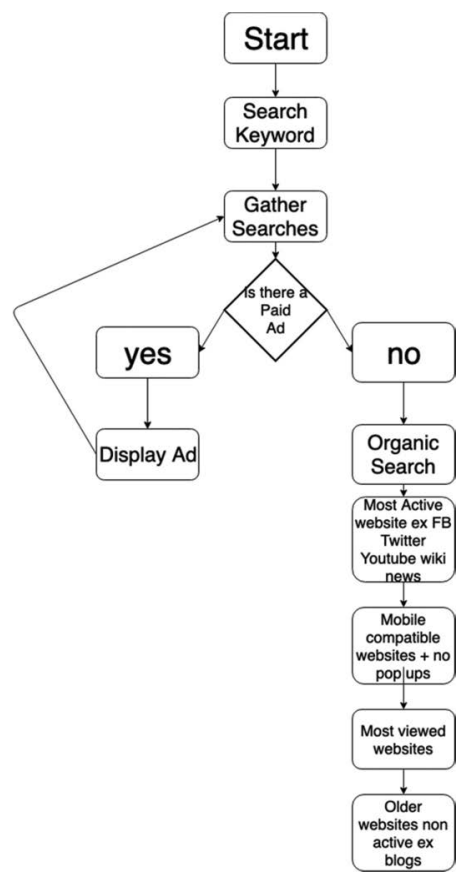

Figure 9 Flowchart of Google algorithm. 
explains the algorithm in simple terms, it can easily be broken down for analysis in the future.

\section{References}

1. AliDropship by Olga L. (2018) 50 Niche Products To Sell In Your Drop Shipping Store In 2019 [Online]. Available: https://alidropship.com/50niche-products-to-sell.

2. Valve Stream. (2019) Steam Community Market [Online]. Available: https://steamcommunity.com/market.

3. Craigslist (2019) Craigslist. [Online]. Available: https://bangkok.craigs list.org/

4. Jonathan Strickland (2007) How Craigslist Works [Online]. Available: https://money.howstuffworks.com/craigslist.htm

5. Money Crashers Suzanne Kearns (2014). 7 Typres of Common Graiglist Scams to Watch Out for [Online]. Available https://www.moneycrashers .com/types-common-craigslist-scams/

6. Chron Luke Arthur (2019). Problems With Craiglist Business Ads. [Online]. Available: https://smallbusiness.chron.com/problemscraigslist-business-ads-27243.html.

7. LifeHacker Adam Dachis (2012) How Can I Avoid Getting Screwed When Selling on Craiglist?. [Online]. Available: https://lifehacker.com/ how-can-i-avoid-getting-screwed-when-selling-on-craigsl-5915608

8. Move To Bangkok (2016) Bangkok Craigslist (and the Best Alternatives) [Online]. Available: https://movetobangkokguide.com/bangkokcraigs list-and-the-best-alternatives/.

9. Craigslists (2019) Craiglists [Online]. Available: https://bangkok.craigs list.org/d/for-sale/search/sss

10. Expat (2019) Chian Mai classifieds [Online]. Available: https://www. expat.com/en/classifieds/asia/thailand/chiang-mai/

11. Craig Newmark Philanthropies (2020) [Online]. Available: https://craign ewmarkphilanthropies.org/

12. Accessify (2019) Page Load Speed Analysis [Online]. Available: https://www.accessify.com/c/bangkok.craigslist.co.th 
13. Craiglists (2019) Craiglists [Online]. Available: https://bangkok.craigs list.org/search/sss?excats

14. World Population Review (2019) Bangkok Population 2019 [Online]. Available: http://worldpopulationreview.com/world-cities/bangkokpopulation/

15. Citypopulation (2020) Bangkok Metropolitan Region [Online]. Available: https://www.citypopulation.de/en/thailand/bangkokmetropolitan/

16. Craigslist (2020) How to Search Craigslist [Online]. Available: https://www.craigslist.org/about/help/search

17. Statista (2019) Number of Facebook users in Thailand from 2017 to 2023 (in Millions) [Online]. Available: https://www.statista.com/statistics 1490467/number-of-thailand-facebook-users/

18. Pricepanda (2019) Pricepanda [Online]. Available: https://www.price panda.co.th/

\section{Biographies}

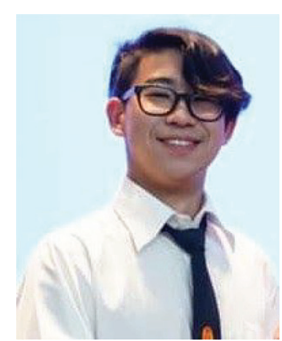

Sutipong Sutipitakwong is a B.Sc. student at Faculty of Information Technology, King Mongkut's Institute of Technology Ladkrabang. He is currently studying in the international programme of Business Information Technology (BIT), at the Faculty of Information Technology. He has received his first conference publication in 2019. Sutipong has been working on several papers including topics regarding; e-learning, ML, and various Technology related researches. 


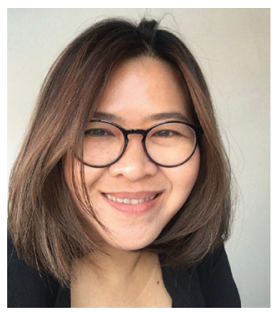

Pornsuree Jamsri is currently teaching at the Faculty of Information Technology, King Mongkut's Institute of Technology Ladkrabang, Bangkok. She received Ph.D. in Information Science \& Learning Technologies from University of Missouri-Columbia, MO; M.Sc. in Human Computer Interaction Design from Indiana University-Bloomington, IN; M.A. in International Human Rights Program from Mahidol University-Salaya, Nakornprathom. She has been working with tech company in the US and Thailand for decades as an UX researcher and technology consultant in various industries including education, health, environment, and agriculture. 